\title{
A NEW ELEMENTARY PROOF FOR M-STATIONARITY UNDER MPCC-GCQ FOR MATHEMATICAL PROGRAMS WITH COMPLEMENTARITY CONSTRAINTS
}

\author{
Felix Harder *
}

\begin{abstract}
It is known in the literature that local minimizers of mathematical programs with complementarity constraints (MPCCs) are so-called M-stationary points, if a weak MPCC-tailored Guignard constraint qualification (called MPCC-GCQ) holds. In this paper we present a new elementary proof for this result. Our proof is significantly simpler than existing proofs and does not rely on deeper technical theory such as calculus rules for limiting normal cones. A crucial ingredient is a proof of a (to the best of our knowledge previously open) conjecture, which was formulated in a Diploma thesis by Schinabeck.
\end{abstract}

Keywords: Mathematical program with complementarity constraints, Necessary optimality conditions, M-stationarity, Guignard constraint qualification

$\operatorname{MSC}(2020): 90 C_{33}, 90 C_{30}$

\section{INTRODUCTION}

We consider mathematical programs with complementarity constraints, or MPCCs for short, which are nonlinear optimization problems of the form

$$
\begin{array}{rl}
\min _{x \in \mathbb{R}^{n}} & f(x) \\
\text { s.t. } & g(x) \leq 0, \quad h(x)=0, \\
& G(x) \geq 0, \quad H(x) \geq 0, \quad G(x)^{\top} H(x)=0 .
\end{array}
$$

Here, $f: \mathbb{R}^{n} \rightarrow \mathbb{R}, g: \mathbb{R}^{n} \rightarrow \mathbb{R}^{l}, h: \mathbb{R}^{n} \rightarrow \mathbb{R}^{m}, G, H: \mathbb{R}^{n} \rightarrow \mathbb{R}^{p}$ are differentiable functions.

MPCCs have been studied extensively in the literature, both from a numerical and a theoretical perspective. Various problem-tailored stationarity conditions and constraint qualifications have been developed. One (necessary, first-order) stationarity condition for MPCCs is the so-called strong stationarity (which is equivalent to the KKT conditions). However, the strong stationarity condition is often too strong, as there are examples of MPCCs where the data is linear, but the unique minimizer is not strongly stationary, see [11, Example 3].

In this article we will focus on M-stationarity (see Definition 2.1), which is another prominent stationarity condition for MPCCs in the literature. We mention that there are other stationarity

The author would like to thank Gerd Wachsmuth for making the author aware of the conjecture in the Diploma thesis [12]. This work is supported by the DFG Grant Bilevel Optimal Control: Theory, Algorithms, and Applications (Grant No. WA 3636/4-2) within the Priority Program SPP 1962 (Non-smooth and Complementarity-based Distributed Parameter Systems: Simulation and Hierarchical Optimization).

* Institute of Mathematics, Chair of Optimal Control. Brandenburgische Technische Universität Cottbus-Senftenberg, Germany.harder@b-tu.de. 
conditions between $\mathrm{M}$ - and strong stationarity, such as extended $\mathrm{M}$-stationarity, strong M-stationarity, $Q_{M^{-}}$stationarity, and linearized $M$-stationarity, see $[6,2,7]$. However, these conditions are used less frequently than $\mathrm{M}$-stationarity and are more complicated to formulate.

As shown in [5], M-stationarity holds for local minimizers of (MPCC) under MPCC-GCQ, see also [4]. MPCC-GCQ, defined in Definition 2.3, is a relatively weak constraint qualification. These proofs for M-stationarity under MPCC-GCQ rely on advanced techniques from variational analysis such as the concept of the so-called limiting normal cone. In particular, calculus rules for limiting normal cones are used, which are based on deeper technical theory and require to verify the calmness of certain set-valued mappings.

We mention that there is also a weaker constraint qualification in $[8,(6)]$, which is the weakest constraint qualification under which local minimizers are M-stationary. An advantage is that the proof of M-stationarity under this constraint qualification is very simple, but on the other hand the proof that MPCC-GCQ implies this constraint qualification (or that this constraint qualification is satisfied for MPCCs with affine data) relies on deeper results from variational analysis. Since MPCC-GCQ and MPCC-ACQ (which trivially implies MPCC-GCQ) are used more frequently, we focus on MPCC-GCQ in this article.

In this paper we want to present a new proof for the result from [5] that M-stationarity holds for local minimizers under MPCC-GCQ. Our new proof does not rely on advanced theory such as the properties of limiting normal cones. Our proof is significantly simpler than any existing proofs that we are aware of. The main ingredient of the new proof is a result which can be found in Lemma 3.2. This result was already conjectured in [12, Section 4.4.2], and, to the best of our knowledge there has not been a proof of this conjecture so far. With the knowledge that Lemma 3.2 holds, the rest of the proof of M-stationarity under MPCC-GCQ will not be particularly surprising for readers familiar with the implications of MPCC-GCQ. For the convenience of the reader we give a self-contained presentation, which only requires familiarity with the basic theory of nonlinear optimization. Since the constraint qualification in $[8,(6)]$ is the weakest constraint qualification under which local minimizers are M-stationary, the new proof also leads to a completely elementary proof that MPCC-GCQ implies $[8,(6)]$.

The structure of this paper is as follows: In Section 2, we introduce classical definitions related to MPCCs. Then we use MPCC-GCQ to construct various multipliers that satisfy some auxiliary stationarity systems, see Proposition 3.1. Afterwards, these multipliers are combined into a multiplier which is M-stationary with the help of Lemma 3.2. The main result is then stated in Theorem 3.3. Finally, we discuss conclusions and perspectives in Section 4.

\section{DEFINITIONS}

It will be convenient to work with the index sets

$$
\begin{aligned}
I^{l} & :=\{1, \ldots, l\}, \quad I^{m}:=\{1, \ldots, m\}, \quad I^{p}:=\{1, \ldots, p\}, \\
I^{g}(\bar{x}) & :=\left\{i \in I^{l} \mid g_{i}(\bar{x})=0\right\}, \\
I^{+0}(\bar{x}) & :=\left\{i \in I^{p} \mid G_{i}(\bar{x})>0 \wedge H_{i}(\bar{x})=0\right\}, \\
I^{0+}(\bar{x}) & :=\left\{i \in I^{p} \mid G_{i}(\bar{x})=0 \wedge H_{i}(\bar{x})>0\right\}, \\
I^{00}(\bar{x}) & :=\left\{i \in I^{p} \mid G_{i}(\bar{x})=0 \wedge H_{i}(\bar{x})=0\right\},
\end{aligned}
$$

where $\bar{x} \in \mathbb{R}^{n}$ is a feasible point of (MPCC). Note that $I^{+0}(\bar{x}), I^{0+}(\bar{x}), I^{00}(\bar{x})$ form a partition of $I^{p}$. We continue with the definition of M-, A- and S-stationarity.

Definition 2.1. Let $\bar{x} \in \mathbb{R}^{n}$ be a feasible point of (MPCC). We call $\bar{x}$ an M-stationary point of (MPCC) if 
there exist multipliers $\bar{\lambda} \in \mathbb{R}^{l}, \bar{\eta} \in \mathbb{R}^{m}, \bar{\mu}, \bar{v} \in \mathbb{R}^{p}$ with

$$
\begin{aligned}
\nabla f(\bar{x})+\sum_{i \in I^{l}} \bar{\lambda}_{i} \nabla g_{i}(\bar{x})+\sum_{i \in I^{m}} \bar{\eta}_{i} \nabla h_{i}(\bar{x})-\sum_{i \in I^{p}}\left(\bar{\mu}_{i} \nabla G_{i}(\bar{x})+\bar{v}_{i} \nabla H_{i}(\bar{x})\right) & =0, \\
\forall i \in I^{g}(\bar{x}): & \bar{\lambda}_{i} \geq 0,
\end{aligned}
$$

$$
\forall i \in I^{g}(\bar{x}):
$$

$\forall i \in I^{l} \backslash I^{g}(\bar{x}):$

$\bar{\lambda}_{i}=0$,

$\forall i \in I^{+0}(\bar{x}):$

$\bar{\mu}_{i}=0$,

$\forall i \in I^{0+}(\bar{x}):$

$\bar{v}_{i}=0$,

$$
\forall i \in I^{00}(\bar{x}): \quad\left(\bar{\mu}_{i}>0 \wedge \bar{v}_{i}>0\right) \vee \bar{\mu}_{i} \bar{v}_{i}=0 .
$$

If the multipliers $\bar{\lambda}, \bar{\eta}, \bar{\mu}, \bar{v}$ only satisfy (2.1a)-(2.1e) and $\bar{\mu}_{i} \geq 0 \vee \bar{v}_{i} \geq 0$ holds for all $i \in I^{00}(\bar{x})$, then $\bar{x}$ is called an A-stationary point of (MPCC). If, additionally, $\bar{\mu}_{i} \geq 0 \wedge \bar{v}_{i} \geq 0$ holds for all $i \in I^{00}(\bar{x})$, then $\bar{x}$ is called a strongly stationary or S-stationary point of (MPCC).

These stationarity conditions can be found in [13, Definitions 2.5-2.7]. Other known stationarity conditions B-, W- and C-stationarity, see [13, Definitions 2.2-2.4].

In preparation for the definition of MPCC-GCQ we define some cones.

Definition 2.2. Let $\bar{x} \in \mathbb{R}^{n}$ be a feasible point of (MPCC).

(a) We define the tangent cone of (MPCC) at $\bar{x}$ via

$$
\mathcal{T}(\bar{x}):=\left\{d \in \mathbb{R}^{n} \mid \begin{array}{r}
\exists\left\{x_{k}\right\}_{k \in \mathbb{N}} \subset F, \exists\left\{t_{k}\right\}_{k \in \mathbb{N}} \subset(0, \infty): \\
x_{k} \rightarrow \bar{x}, t_{k} \downarrow 0, t_{k}^{-1}\left(x_{k}-\bar{x}\right) \rightarrow d
\end{array}\right\},
$$

where $F \subset \mathbb{R}^{n}$ denotes the feasible set of (MPCC).

(b) We define the MPCC-linearized tangent cone $\mathcal{T}_{\mathrm{MPCC}}^{\operatorname{lin}}(\bar{x}) \subset \mathbb{R}^{n}$ at $\bar{x}$ via

$$
\mathcal{T}_{\mathrm{MPCC}}^{\operatorname{lin}}(\bar{x}):=\left\{d \in \mathbb{R}^{n} \begin{array}{rl}
\nabla g_{i}(\bar{x})^{\top} d \leq 0 & \forall i \in I^{g}(\bar{x}), \\
\nabla h_{i}(\bar{x})^{\top} d=0 & \forall i \in I^{m}, \\
\nabla G_{i}(\bar{x})^{\top} d=0 & \forall i \in I^{0+}(\bar{x}), \\
\nabla H_{i}(\bar{x})^{\top} d=0 & \forall i \in I^{+0}(\bar{x}), \\
\nabla G_{i}(\bar{x})^{\top} d \geq 0 & \forall i \in I^{00}(\bar{x}), \\
\nabla H_{i}(\bar{x})^{\top} d \geq 0 & \forall i \in I^{00}(\bar{x}), \\
\left(\nabla G_{i}(\bar{x})^{\top} d\right)\left(\nabla H_{i}(\bar{x})^{\top} d\right)=0 & \forall i \in I^{00}(\bar{x})
\end{array}\right\} .
$$

Note that in general $\mathcal{T}(\bar{x})$ and $\mathcal{T}_{\text {MPCC }}^{\operatorname{lin}}(\bar{x})$ are nonconvex cones.

We also recall that the polar cone $C^{\circ}$ of a set $C \subset \mathbb{R}^{n}$ is defined via

$$
C^{\circ}:=\left\{d \in \mathbb{R}^{n} \mid d^{\top} y \leq 0 \quad \forall y \in C\right\} .
$$

Now we are ready to give the definition of MPCC-GCQ, which can also be found in [5, (41)], where it is called MPEC-GCQ.

Definition 2.3. Let $\bar{x} \in \mathbb{R}^{n}$ be a feasible point of (MPCC). We say that $\bar{x}$ satisfies the MPCC-tailored Guignard constraint qualification, or MPCC-GCQ, if

$$
\mathcal{T}(\bar{x})^{\circ}=\mathcal{T}_{\text {MPCC }}^{\operatorname{lin}}(\bar{x})^{\circ}
$$

holds. Additionally, if $\mathcal{T}(\bar{x})=\mathcal{T}_{\text {MPCC }}^{\operatorname{lin}}(\bar{x})$ holds then we say that $\bar{x}$ satisfies MPCC-ACQ.

Clearly, MPCC-ACQ implies MPCC-GCQ. We mention that there are also other stronger constraint qualifications (such as MPCC-MFCQ if $g, h, G, H$ are continuously differentiable, defined in [3, Definition 2.1]) which imply MPCC-ACQ or MPCC-GCQ and are sometimes easier to verify, see e.g. [13, Theorem 3.2]. In particular, we emphasize that MPCC-GCQ (and MPCC-ACQ) are satisfied at every feasible point of (MPCC) if the functions $g, h, G, H$ are affine, see [3, Theorem 3.2]. 


\section{M-STATIONARITY UNDER MPCC-GCQ}

We start with a proposition that generates several multipliers which satisfy a slightly stronger stationarity condition than A-stationarity. The result can also be obtained from the proof of [3, Theorem 3.4], with the minor difference that we only require MPCC-GCQ and not MPCC-ACQ.

Proposition 3.1. Let $\bar{x} \in \mathbb{R}^{n}$ be a local minimizer of (MPCC) that satisfies MPCC-GCQ and let $\alpha \in\{1,2\}^{p}$ be given. Then there exist multipliers $\lambda^{\alpha} \in \mathbb{R}^{l}, \eta^{\alpha} \in \mathbb{R}^{m}, \mu^{\alpha}, v^{\alpha} \in \mathbb{R}^{p}$ with

$$
\nabla f(\bar{x})+\sum_{i \in I^{l}} \lambda_{i}^{\alpha} \nabla g_{i}(\bar{x})+\sum_{i \in I^{m}} \eta_{i}^{\alpha} \nabla h_{i}(\bar{x})-\sum_{i \in I^{p}}\left(\mu_{i}^{\alpha} \nabla G_{i}(\bar{x})+v_{i}^{\alpha} \nabla H_{i}(\bar{x})\right)=0
$$

$$
\begin{array}{ll}
\forall i \in I^{g}(\bar{x}): & \lambda_{i}^{\alpha} \geq 0, \\
\forall i \in I^{l} \backslash I^{g}(\bar{x}): & \lambda_{i}^{\alpha}=0, \\
\forall i \in I^{+0}(\bar{x}): & \mu_{i}^{\alpha}=0, \\
\forall i \in I^{0+}(\bar{x}): & v_{i}^{\alpha}=0, \\
\forall i \in I^{00}(\bar{x}), \alpha_{i}=1: & \mu_{i}^{\alpha} \geq 0, \\
\forall i \in I^{00}(\bar{x}), \alpha_{i}=2: & v_{i}^{\alpha} \geq 0 .
\end{array}
$$

Proof. From Definition 2.2 (a) and the fact that $\bar{x}$ is a local minimizer of (MPCC) it can be concluded that the condition

$$
\nabla f(\bar{x})^{\top} d \geq 0 \quad \forall d \in \mathcal{T}(\bar{x})
$$

is satisfied. Using polar cones and MPCC-GCQ, we obtain

$$
-\nabla f(\bar{x}) \in \mathcal{T}(\bar{x})^{\circ}=\mathcal{T}_{\text {MPCC }}^{\operatorname{lin}}(\bar{x})^{\circ} .
$$

Furthermore, we define the cone $\mathcal{T}_{\mathrm{NLP}(\alpha)}^{\operatorname{lin}}(\bar{x}) \subset \mathcal{T}_{\mathrm{MPCC}}^{\operatorname{lin}}(\bar{x})$ via

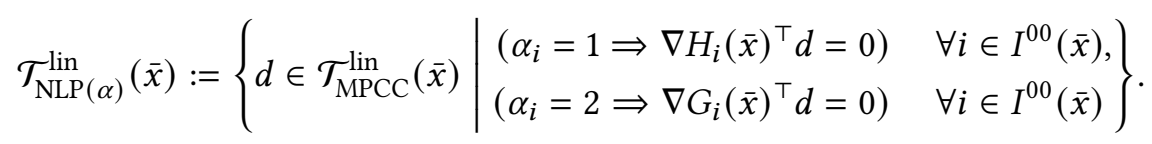

Using the definition of polar cones, the inclusion $\mathcal{T}_{\mathrm{MPCC}}^{\operatorname{lin}}(\bar{x})^{\circ} \subset \mathcal{T}_{\mathrm{NLP}(\alpha)}^{\operatorname{lin}}(\bar{x})^{\circ}$ follows from $\mathcal{T}_{\mathrm{NLP}(\alpha)}^{\operatorname{lin}}(\bar{x}) \subset$ $\mathcal{T}_{\mathrm{MPCC}}^{\operatorname{lin}}(\bar{x})$. In particular, we have $-\nabla f(\bar{x}) \in \mathcal{T}_{\mathrm{NLP}(\alpha)}^{\operatorname{lin}}(\bar{x})^{\circ}$. Note that the condition $\left(\nabla G_{i}(\bar{x})^{\top} d\right)\left(\nabla H_{i}(\bar{x})^{\top} d\right)=$ $0 \forall i \in I^{00}(\bar{x})$ from $\mathcal{T}_{\mathrm{MPCC}}^{\operatorname{lin}}(\bar{x})$ is redundant in $\mathcal{T}_{\mathrm{NLP}(\alpha)}^{\operatorname{lin}}(\bar{x})$, and therefore $\mathcal{T}_{\mathrm{NLP}(\alpha)}^{\operatorname{lin}}(\bar{x})$ is a convex and polyhedral cone (unlike $\mathcal{T}_{\text {MPCC }}^{\operatorname{lin}}(\bar{x})$ ). Thus, one can calculate its polar cone (e.g. using Farkas' Lemma), which results in

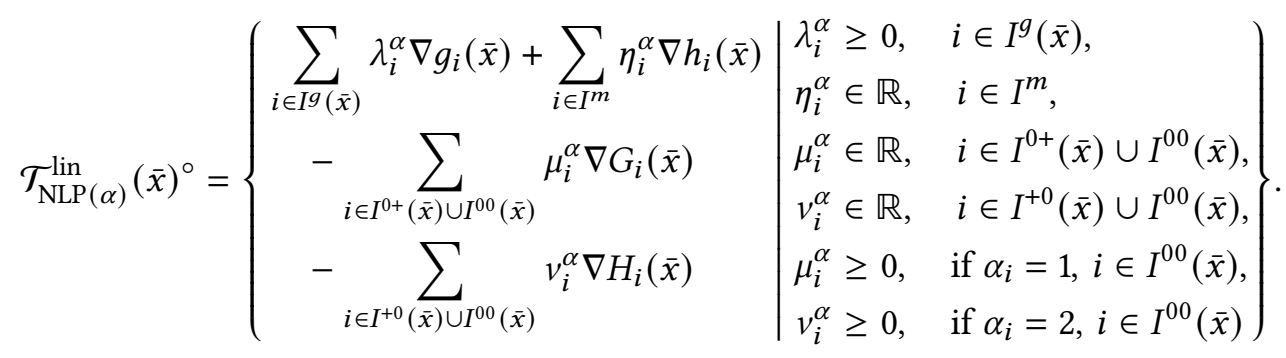

Then the result follows from $-\nabla f(\bar{x}) \in \mathcal{T}_{\operatorname{NLP}(\alpha)}^{\operatorname{lin}}(\bar{x})^{\circ}$ by setting the remaining components of the multipliers (i.e. $\lambda_{i}^{\alpha}$ for $i \in I^{p} \backslash I^{g}(\bar{x}), \mu_{i}^{\alpha}$ for $i \in I^{+0}(\bar{x})$, $v_{i}^{\alpha}$ for $\left.i \in I^{0+}(\bar{x})\right)$ to zero.

We mention that if $\bar{x}$ satisfies (3.1) for some $\alpha \in\{1,2\}^{p}$ and suitable multipliers, then $\bar{x}$ is an Astationary point of (MPCC). However, the statement of Proposition 3.1 is stronger than A-stationarity, 
namely for each index in $I^{00}(\bar{x})$ we can choose whether $\mu_{i}^{\alpha}$ or $v_{i}^{\alpha}$ is nonnegative. Note that (2.1a)-(2.1e) are already satisfied by all $2^{p}$ possible choices for the multipliers and any convex combination of these. Thus, the question naturally arises whether a convex combination of these multipliers can be found that also satisfies (2.1f). As the next result shows, this is indeed possible. The following lemma was already stated as a conjecture in [12, Section 4.4.2]. To the best of our knowledge, this conjecture has not been proven before.

Lemma 3.2. Let $\hat{I} \subset I^{p}$ be an index set. For all $\alpha \in\{1,2\}^{p}$, let points $\left(\mu^{\alpha}, v^{\alpha}\right) \in A^{\alpha}$ be given, where the set $A^{\alpha}$ is described via

$$
A^{\alpha}:=\left\{(\mu, v) \in \mathbb{R}^{2 p} \mid \mu_{i} \geq 0 \text { if } \alpha_{i}=1, v_{i} \geq 0 \text { if } \alpha_{i}=2 \quad \forall i \in \hat{I}\right\} .
$$

Then there exists a point $(\bar{\mu}, \bar{v})$ in the set

$$
B:=\operatorname{conv}\left\{\left(\mu^{\alpha}, v^{\alpha}\right) \mid \alpha \in\{1,2\}^{p}\right\} \subset \mathbb{R}^{2 p}
$$

of convex combinations of these points, such that for all $i \in \hat{I}$ we have the condition

$$
\left(\bar{\mu}_{i}>0 \wedge \bar{v}_{i}>0\right) \vee \bar{\mu}_{i} \bar{v}_{i}=0 .
$$

Proof. Let us choose points $\left(\hat{\mu}^{\alpha}, \hat{v}^{\alpha}\right) \in B \cap A^{\alpha}$, a vector $\beta \in\{1,2\}^{p}$, and points $\bar{\mu}, \bar{v} \in \mathbb{R}^{p}$ that satisfy

$$
\begin{gathered}
\left(\hat{\mu}^{\alpha}, \hat{v}^{\alpha}\right) \in \underset{(\mu, v) \in B \cap A^{\alpha}}{\arg \min }\|(\mu, v)\|_{2}^{2} \quad \forall \alpha \in\{1,2\}^{p}, \\
\beta \in \underset{\alpha \in\{1,2\}^{p}}{\arg \max }\left\|\left(\hat{\mu}^{\alpha}, \hat{v}^{\alpha}\right)\right\|_{2}^{2}, \\
(\bar{\mu}, \bar{v}):=\left(\hat{\mu}^{\beta}, \hat{v}^{\beta}\right) \in \mathbb{R}^{2 p} .
\end{gathered}
$$

These choices are possible because the sets $B \cap A^{\alpha}$ is compact and nonempty (the nonemptiness follows from $\left.\left(\mu^{\alpha}, v^{\alpha}\right) \in B \cap A^{\alpha}\right)$ and the set $\{1,2\}^{p}$ is finite. Furthermore, we have $(\bar{\mu}, \bar{v}) \in B$, i.e. it is a convex combination as claimed.

It remains to show that our choice $\left(\hat{\mu}^{\beta}, \hat{v}^{\beta}\right)$ for $(\bar{\mu}, \bar{v})$ satisfies (3.2). By contradiction, we assume that there exists an $i \in \hat{I}$ such that (3.2) is not satisfied, i.e. $\hat{\mu}_{i}^{\beta} \neq 0, \hat{v}_{i}^{\beta} \neq 0$, and $\hat{\mu}_{i}^{\beta}<0 \vee \hat{v}_{i}^{\beta}<0$ hold. Without loss of generality we can assume that $\beta_{i}=1$ holds (otherwise one would exchange the roles of $\mu$ and $v$ in the rest of the proof). Therefore, we have $\hat{\mu}_{i}^{\beta} \geq 0$ due to $\left(\hat{\mu}^{\beta}, \hat{v}^{\beta}\right) \in A^{\beta}$. Since (3.2) is not satisfied this implies $\hat{\mu}_{i}^{\beta}>0$ and $\hat{v}_{i}^{\beta}<0$. We define

$$
\gamma \in\{1,2\}^{p}, \quad \gamma_{j}:=\left\{\begin{array}{ll}
2 & \text { if } j=i, \\
\beta_{j} & \text { if } j \in I^{p} \backslash\{i\}
\end{array} \quad \forall j \in I^{p} .\right.
$$

Due to $\hat{\mu}_{i}^{\beta}>0$ we can choose $t \in(0,1)$ such that the convex combination

$$
\left(\mu^{t}, v^{t}\right):=t\left(\hat{\mu}^{\gamma}, \hat{v}^{\gamma}\right)+(1-t)\left(\hat{\mu}^{\beta}, \hat{v}^{\beta}\right) \in \mathbb{R}^{2 p}
$$

still satisfies $\mu_{i}^{t}>0$. Since $\gamma_{j}=\beta_{j}$ holds for $j \neq i$ we also have $\left(\mu^{t}, v^{t}\right) \in A^{\beta} \cap B$. However, $\hat{v}_{i}^{\gamma} \geq 0$ implies $\left(\hat{\mu}^{\beta}, \hat{v}^{\beta}\right) \neq\left(\hat{\mu}^{\gamma}, \hat{v}^{\gamma}\right)$. Thus, by also using (3.4), we have

$$
\left\|\left(\mu^{t}, v^{t}\right)\right\|_{2}^{2}<\max \left\{\left\|\left(\hat{\mu}^{\beta}, \hat{v}^{\beta}\right)\right\|_{2}^{2},\left\|\left(\hat{\mu}^{\gamma}, \hat{v}^{\gamma}\right)\right\|_{2}^{2}\right\}=\left\|\left(\hat{\mu}^{\beta}, \hat{v}^{\beta}\right)\right\|_{2}^{2}
$$

Due to $\left(\mu^{t}, v^{t}\right) \in B \cap A^{\beta}$ this is a contradiction to (3.3), which completes the proof. 
Instead of the function $(\mu, v) \mapsto\|(\mu, v)\|_{2}^{2}$, any strictly convex function on $\mathbb{R}^{2 p}$ would have worked in the proof.

We mention that it was recognized already in [12, Section 4.4.2] that this lemma would significantly simplify the already existing proofs for M-stationarity. We further mention that the idea to combine various multipliers of the form $\left(\mu^{\alpha}, v^{\alpha}\right)$ was also used for the concept of $Q$-stationarity in [1].

A straightforward combination of Proposition 3.1 and Lemma 3.2 yields the desired M-stationarity result.

Theorem 3.3. Let $\bar{x} \in \mathbb{R}^{n}$ be a local minimizer of (MPCC) that satisfies MPCC-GCQ. Then $\bar{x}$ is an M-stationary point.

Proof. For all $\alpha \in\{1,2\}^{p}$, let $\left(\lambda^{\alpha}, \eta^{\alpha}, \mu^{\alpha}, v^{\alpha}\right) \in \mathbb{R}^{l+m+2 p}$ be the multipliers generated by Proposition 3.1. By applying Lemma 3.2 with $\hat{I}=I^{00}(\bar{x})$, we find a convex combination $(\bar{\lambda}, \bar{\eta}, \bar{\mu}, \bar{v}) \in \mathbb{R}^{l+m+2 p}$ of these multipliers such that (2.1f) is satisfied. The conditions (2.1a)-(2.1e) follow from (3.1a)-(3.1e) by convexity.

\section{CONCLUSION AND OUTLOOK}

We provided a new proof for the M-stationarity of local minimizers of MPCCs under MPCC-GCQ. Although this result was already known, the new proof uses only basic and well-known tools from nonlinear programming theory. This new elementary proof for M-stationarity was enabled by proving a (to the best of our knowledge previously open) conjecture from [12] in Lemma 3.2.

In the future, it would also be interesting to apply this approach to other problem classes from disjunctive programming and to investigate to what extend the ideas from this paper can be generalized.

In Sobolev or Lebesgue spaces, the limiting normal cone turned out to be not as effective as in finite dimensional spaces for obtaining stationarity conditions for complementarity-type optimization problems, see $[9,10]$. Thus, it would be interesting to know whether the new elementary method from this paper can provide ideas for possible approaches for better stationarity conditions of complementaritytype optimization problems in Sobolev and Lebesgue spaces.

\section{REFERENCES}

[1] M. Benko and H. Gfrerer, On estimating the regular normal cone to constraint systems and stationarity conditions, Optimization 66 (2016), 61-92, doi:10.1080/02331934.2016.1252915.

[2] M. Benko and H. Gfrerer, New verifiable stationarity concepts for a class of mathematical programs with disjunctive constraints, Optimization 67 (2017), 1-23, doi:10.1080/02331934.2017.1387547.

[3] M. L. Flegel and C. Kanzow, Abadie-type constraint qualification for mathematical programs with equilibrium constraints, fournal of Optimization Theory and Applications 124 (2005), 595-614, doi:10.1007/s10957-004-1176-X.

[4] M. L. Flegel and C. Kanzow, A direct proof for M-stationarity under MPEC-GCQ for mathematical programs with equilibrium constraints, in Optimization with Multivalued Mappings, volume 2 of Springer Optim. Appl., Springer, New York, 2006, 111-122, doi:10.1007/0-387-34221-4_6.

[5] M. L. Flegel, C. Kanzow, and J.V. Outrata, Optimality conditions for disjunctive programs with application to mathematical programs with equilibrium constraints, Set-Valued Analysis 15 (2007), 139-162, doi:10.1007/s11228-006-0033-5.

[6] H. Gfrerer, Optimality conditions for disjunctive programs based on generalized differentiation with application to mathematical programs with equilibrium constraints, SIAM fournal on Optimization 24 (2014), 898-931, doi:10.1137/130914449. 
[7] H. Gfrerer, Linearized M-stationarity conditions for general optimization problems, Set-Valued and Variational Analysis 27 (2018), 819-840, doi:10.1007/s11228-018-0491-6.

[8] L. Guo and G. H. Lin, Notes on some constraint qualifications for mathematical programs with equilibrium constraints, fournal of Optimization Theory and Applications 156 (2012), 60o-616, doi:10.1007/s10957-012-0084-8.

[9] F. Harder and G. Wachsmuth, The limiting normal cone of a complementarity set in Sobolev spaces, Optimization 67 (2018), 1579-1603, doi:10.1080/02331934.2018.1484467.

[10] P. Mehlitz and G. Wachsmuth, The limiting normal cone to pointwise defined sets in Lebesgue spaces, Set-Valued and Variational Analysis 26 (2018), 449-467, doi:10.1007/s11228-016-0393-4.

[11] S. Scheel and S. Scholtes, Mathematical programs with complementarity constraints: stationarity, optimality, and sensitivity, Mathematics of Operations Research 25 (2000), 1-22, doi:10.1287/moor. 25.1.1.15213.

[12] M. Schinabeck, Constraint Qualifications und Optimalitätsbedingungen für MPECs, Diploma thesis, University of Regensburg, Germany, 2009.

[13] J.J. Ye, Necessary and sufficient optimality conditions for mathematical programs with equilibrium constraints, Journal of Mathematical Analysis and Applications 307 (2005), 350 - 369, doi:10.1016/j. jmaa.2004.10.032. 